\title{
Singularities of Corner Problems and Problems of Corner Singularities
}

\author{
Monique DAUGE
}

Résumé. Dans le cadre des problèmes aux limites elliptiques du second ordre, nous présentons l'essentiel de la structure des solutions singulières auprès des sommets et arêtes d'un polyèdre, et nous évoquons les difficultés inhérentes à la présence de ces singularités.

Mots clés. Coin, Arête, Sommet, Problème aux limites elliptique, Singularité.

Abstract. In the framework of second order elliptic boundary value problems, we describe the main features of the singular solutions near vertices and edges of a polyhedron, and we discuss some difficulties arising from the presence of these singularities.

Keywords. Corner, Edge, Vertex, Elliptic Boundary Value Problem, Singularities.

AMS Subject Classification. $35 \mathrm{~J}, 65 \mathrm{~N}, 73 \mathrm{C}$

\section{Non-smooth domains and domains with corners}

At our natural observation scale, "corners" are everywhere: they belong to sharp or reentrant edges, to cracks fronts, they are also the meeting points of several edges. At a micro industrial scale, the constituting parts of electronic components have many edges and sharp corners, combined moreover with very large (or small) aspect ratios. At the material structure scale, alloys and ceramic defaults look like micro-cracks. But, at
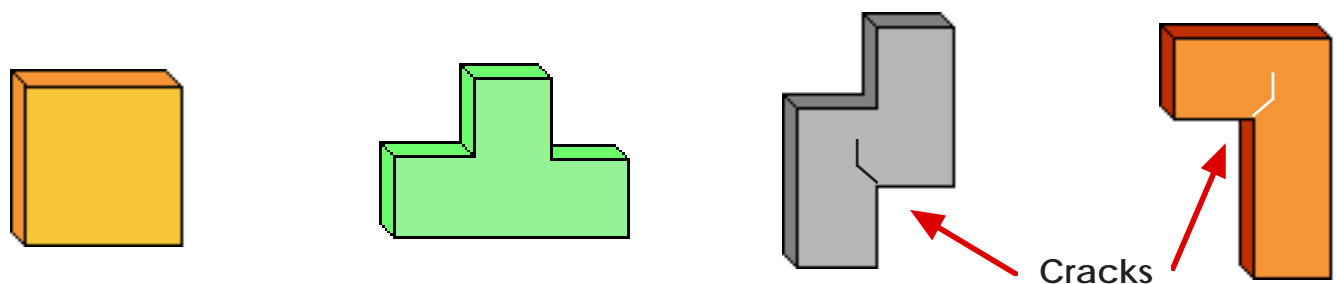

Figure 1: Polyhedral domains and cracks

the molecular scale, the boundaries of a domain are meaningless: faces, edges or vertices cannot be "seen" at this scale.

Article published by EDP Sciences and available at http://www.edpsciences.org/proc or http://dx.doi.org/10.1051/proc:1999044 
A mathematical domain is an open subset in $\mathbb{R}^{n}$ where partial differential equations are set, and represent an idealized subset of the physical world. A smooth domain is then a domain whose boundary can be described by a finite number of smooth maps. Thus a non-smooth domain can have infinitely various descriptions. Here, we will concentrate on polyhedral geometries, letting aside purely Lipschitz domains and domains with cusps. A Lipschitz domain is described by bi-Lipschitz maps and can be very pathologic, containing an infinite number of sides and corners for example. For cuspidal domains (where there are piecewise smooth faces, but tangent in certain points), we refer to [35], [17], [37, 38].

So, to fix ideas, we consider three-dimensional polyhedral domains $\Omega$. To such a domain belong faces $F$, edges $E$ and vertices $\mathbf{v}$, where $\Omega$ is locally diffeomorphic to a half-space $\mathbb{R}^{2} \times \mathbb{R}_{+}$, a wedge $\mathbb{R} \times \Gamma_{E}$ or a polyhedral cone $\Gamma_{\mathbf{v}}$ respectively. The wedge is the product of $\mathbb{R}$ by a plane sector $\Gamma_{E}$ and the polyhedral cone $\Gamma_{\mathbf{v}}$ is an infinite cone with vertex 0 and plane faces.

We end this section by the following remark: in general, polyhedral domains are the limit case of real domains where edges and corners are replaced with neighboring regions where the curvature of the boundary is very large. Though being in principle smooth domains, these more realistic geometries will create at least similar difficulties than polyhedra, see Maz'ya, Nazarov, Plamenevskit [33].

\section{Elliptic boundary value problems}

Here, we do not consider time-dependent problems and we only quote as selection in the literature KozLOV [25] for parabolic problems, and LEBEAU [30] for hyperbolic problems.

We concentrate on steady or periodic states, governed for example by continuum mechanics, fluid mechanics or electromagnetics. In a first approximation, the equations arising from physical laws in these situations can be written as a linear elliptic boundary value problem, and, even, as a coercive variational problem.

Thus, to fix ideas, we consider elliptic boundary value problems generated by a variational formulation based on a subspace $V$ of $H^{1}(\Omega)$ and a coercive integro-differential form $a$ of order 1 :

$$
u \in V, \quad \forall v \in V, \quad a(u, v)=f(v) .
$$

Here are our basic assumptions

- $V=\left\{v \in H^{1}(\Omega) ; v=0\right.$ on $\left.\partial_{D} \Omega\right\}$, where $\partial_{D} \Omega$ is a part of $\partial \Omega$. We assume that the intersection of $\partial_{D} \Omega$ with each face $F$ of $\partial \Omega$ is either empty, or the whole face or a polygonal subset of $F$.

- The form $a$ can be written as

$$
a(u, v)=\sum_{|\alpha| \leq 1} \sum_{|\beta| \leq 1} \int_{\Omega} a_{\alpha \beta}(x) \partial_{x}^{\alpha} u \partial_{x}^{\beta} v d x
$$

where the coefficients $a_{\alpha \beta}$ are "polyhedral piecewise smooth" in the sense that there exists a finite polyhedral partition $\left(\Omega_{j}\right)_{j \in J}$ of $\Omega$ such that all $a_{\alpha \beta}$ are $\mathcal{C}^{\infty}\left(\bar{\Omega}_{j}\right), j \in J$. 
- The right hand side $f$ is a continuous bilinear form on $V$.

The assumption of coerciveness is that there exists $c>0$ such that for all $x \in \bar{\Omega}$ and all $\xi \in \mathbb{R}^{3}$ there holds

$$
\sum_{|\alpha| \leq 1} \sum_{|\beta| \leq 1} a_{\alpha \beta}(x) \xi^{\alpha} \xi^{\beta} \geq c|\xi|^{2} .
$$

Thus the boundary value problem corresponding to (1) is a transmission problem (if we make the more usual assumption that the coefficients $a_{\alpha \beta}$ are smooth on $\bar{\Omega}$, we obtain the boundary value problem consisting in the first two lines of (2))

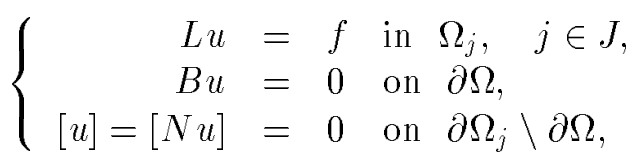

where

- the operator $L$ is equal to $-\partial^{\beta} a_{\alpha \beta}(x) \partial_{x}^{\alpha}$ in each $\Omega_{j}$,

- the boundary operator $B$ is the Dirichlet operator (simple trace) in $\partial_{D} \Omega$ and the Neumann operator $N$ associated with $a$ in $\partial \Omega \backslash \partial_{D} \Omega$,

- the expressions $[u]$ and $[N u]$ are the jumps of $u$ and $N u$ across the interior interfaces contained in $\partial \Omega_{j} \backslash \partial \Omega$. Note that the expressions of $N$ are in general different at each side of an interface.

Examples for such problems are

1. The equation for an electric potential in an heterogeneous material $\left(\varepsilon_{j}\right.$ is the electric permittivity of its homogeneous part $\Omega_{j}$ ) associated with the bilinear form

$$
a(u, v)=\sum_{j} \int_{\Omega_{j}} \varepsilon_{j} \nabla u \cdot \nabla v d x, \quad \text { for } \quad u, v \in H_{0}^{1}(\Omega) .
$$

Here $N=\varepsilon_{j} \partial_{n}$, with $n$ a unit normal derivative on $F$.

2. The equation for the displacement of an elastic multi-material structure ( $A_{j}$ is the rigidity matrix of the material $\Omega_{j}$ ) associated with the bilinear form

$$
a(u, v)=\sum_{j} \int_{\Omega_{j}} A_{j} e(u): e(v) d x, \quad \text { for } \quad u, v \in V,
$$

with $e$ the strain tensor and : indicates the contraction of tensors. Here $N$ is the traction operator $\sigma_{j} \cdot n$, where $\sigma_{j}=A_{j} e(u)$. The part $\partial_{D} \Omega$ is the clamped part of the structure.

Indeed, this framework can be widely extended (general Agmon-Douglis-Nirenberg's type [1] transmission problem of arbitrary order, with a coerciveness assumption) so that there holds similar results to those we will describe. See also Grisvard [20] for oblique derivatives problems in polygons, to NICAISE [41] for polygonal transmission problems and to Dauge, Nicaise [18] for a combination of both. 


\section{Problematics}

In any subdomain $\Omega^{\prime}$ such that $\overline{\Omega^{\prime}}$ is contained in one of the $\Omega_{j}$, the elliptic regularity holds for solutions of problem (1) (shift theorem). That means that if $f$ belongs to $H^{s-1}(\Omega)$ with $s>0$, then $u$ belongs to $H^{s+1}\left(\Omega^{\prime}\right)$. Such a regularity result still holds if $\bar{\Omega}^{\prime}$ intersects $\partial \Omega$ and the $\partial \Omega_{j}$ only in their smooth parts. Then, if $f$ is piecewise $H^{s-1}$, i.e. if

$$
\left.f\right|_{\Omega_{j}} \in H^{s-1}\left(\Omega_{j}\right), \quad \forall j \in J, \quad \text { denoted by } f \in P H^{s-1}(\Omega),
$$

then $u \in P H^{s+1}\left(\Omega^{\prime}\right)$. Now, if $\bar{\Omega}^{\prime}$ intersects any edge or vertex of $\Omega$ or of one of the $\Omega_{j}$, such a result does not hold anymore in general. We will see later on that it holds for small enough $s$, the upper bound being explicit in a certain sense.

Let us point out that for Lipschitz domains and the Dirichlet or Neumann boundary value problems associated with the standard Laplace operator the general bound is known: this is $\frac{1}{2}$, see $[22,23]$. Of course such a result is coherent with what can be obtained for polyhedral domains. But the corresponding bound can be much smaller as soon as transmission problems are concerned.

The determination of the upper bound for regularity is closely related to the spectrum of what we call Mellin symbols along edges and at vertices. Moreover, the eigenfunctions of these symbols generate the singular functions which are precisely the default for the shift theorem to hold.

\section{Mellin symbols}

For the transmission problem (2) associated with the partition $\left(\Omega_{j}\right)_{j \in J}$ the set of vertices is the union of the vertices of all $\Omega_{j}$ and the set of edges is the union of the edges of all $\Omega_{j}$. Of course these sets reduce to the vertices and edges of $\Omega$ respectively if there is no transmission condition inside $\Omega$ and if there is no transition of boundary conditions inside the faces of $\Omega$.

\subsection{Vertices}

Let $\mathbf{v}$ be a vertex in the previous sense. We assume that there exists a local chart which transforms in a neighborhood of $\mathbf{v}, \Omega$ and the $\Omega_{j}$ having $\mathbf{v}$ as vertex into polyhedral cones $\Gamma_{\mathbf{v}}$ and $\Gamma_{\mathbf{v}, j}$. By this local chart, problem (2) is transformed into a similar problem in the cone $\Gamma_{\mathbf{v}}$. In polar coordinates $(\rho, \vartheta)$, the operators $L, B$ and $N$ can be formally expanded in increasing powers of $\rho$ according to:

$$
\left\{\begin{array}{l}
L=\rho^{-2} \mathcal{L}\left(\vartheta ; \rho \partial_{\rho}, \partial_{\vartheta}\right)+\sum_{p \geq 1} \rho^{-2+p} \mathcal{L}_{p}\left(\vartheta ; \rho \partial_{\rho}, \partial_{\vartheta}\right) \\
B=\rho^{-\operatorname{deg} B} \mathcal{B}\left(\vartheta ; \rho \partial_{\rho}, \partial_{\vartheta}\right)+\sum_{p \geq 1} \rho^{-\operatorname{deg} B+p} \mathcal{B}_{p}\left(\vartheta ; \rho \partial_{\rho}, \partial_{\vartheta}\right) \\
N=\rho^{-1} \mathcal{N}\left(\vartheta ; \rho \partial_{\rho}, \partial_{\vartheta}\right)+\sum_{p \geq 1} \rho^{-1+p} \mathcal{N}_{p}\left(\vartheta ; \rho \partial_{\rho}, \partial_{\vartheta}\right)
\end{array}\right.
$$




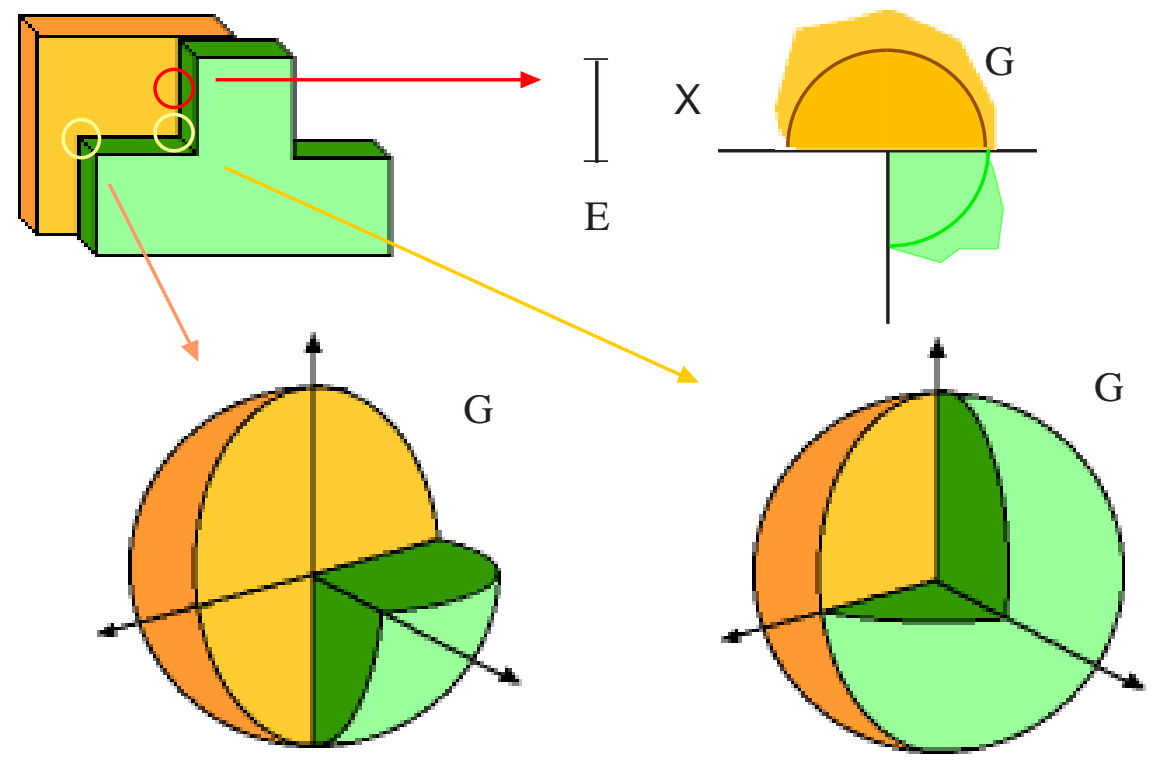

Figure 2: Associated solid angles

To the operators $\mathcal{L}, \mathcal{B}$ and $\mathcal{N}$ are associated their Mellin symbols

$$
\left\{\begin{array}{lll}
\mathbb{C} \ni \lambda & \longmapsto & \mathcal{L}\left(\vartheta ; \lambda, \partial_{\vartheta}\right)=: \mathcal{L}(\lambda) \\
\mathbb{C} \ni \lambda & \longmapsto & \mathcal{B}\left(\vartheta ; \lambda, \partial_{\vartheta}\right)=: \mathcal{B}(\lambda) \\
\mathbb{C} \ni \lambda & \longmapsto & \mathcal{N}\left(\vartheta ; \lambda, \partial_{\vartheta}\right)=: \mathcal{N}(\lambda) .
\end{array}\right.
$$

The denomination "Mellin symbols" comes from the Mellin transform

$$
\mathcal{M}[u](\lambda)=\int_{0}^{\infty} \rho^{-\lambda} u(\rho, \vartheta) \frac{d \rho}{\rho}
$$

which satisfies

$$
\mathcal{M}[\mathcal{L} u](\lambda)=\mathcal{L}(\lambda) \mathcal{M}[u](\lambda)
$$

Let $G_{\mathbf{v}}$ be the solid angle intercepted by $\Gamma_{\mathbf{v}}$ on the sphere $\mathbb{S}^{2}$ (so that there holds $\Gamma_{\mathbf{v}}=$ $\left\{x \in \mathbb{R}^{3} ; \vartheta \in G_{\mathbf{v}}\right\}$ ). Then the operator valued Mellin symbol (also called operator pencil in the Russian literature) associated with problem (2) at the vertex $\mathbf{v}$ is the underlying operator to problem

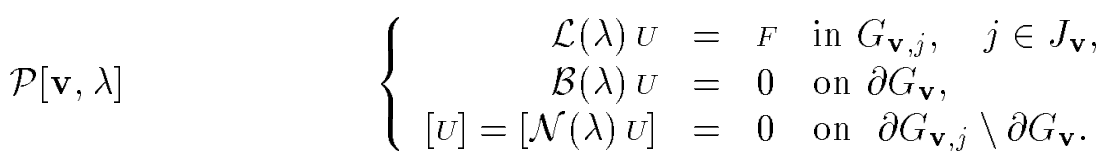

Here $G_{\mathbf{v}, j}$ is the part of $G$ intercepted by the sub-cone $\Gamma_{\mathbf{v}, j}$ corresponding to the subdomain $\Omega_{j}$ for any $j$ such that $\mathbf{v}$ is a vertex of $\Omega_{j}$ (which defines the subset $J_{\mathbf{v}}$ of $J$ ).

The fundamental result concerning the operator valued Mellin symbol is the following consequence of the coerciveness of problem (1), see [16]. 


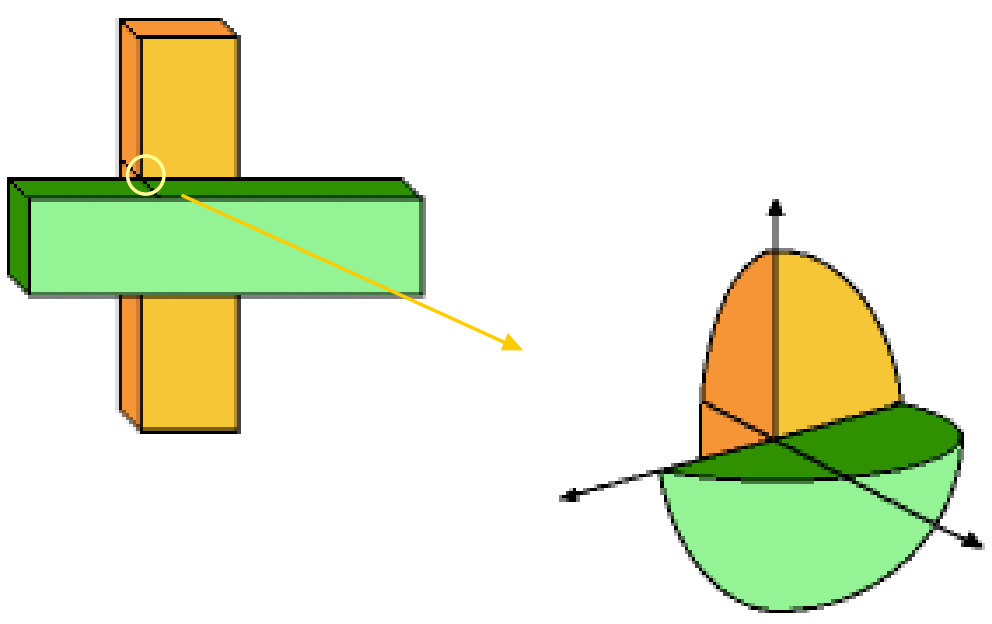

Figure 3: Associated solid angle to a non-Lipschitz corner

Lemma 4.1 Problem $\mathcal{P}[\mathbf{v}, \lambda]$ is uniquely solvable in $H^{1}\left(G_{\mathbf{v}}\right)$ for all $\lambda \in \mathbb{C} \backslash \mathcal{S}(\mathbf{v})$ where $\mathcal{S}[\mathbf{v}]$ is a discrete set (the spectrum). The set $\mathcal{S}[\mathbf{v}]$ has the following property: in all strip of the form $\operatorname{Re} \lambda \in[a, b]$, with $a, b \in \mathbb{R}$, the number of elements of $\mathcal{S}[\mathbf{v}]$ is finite. Moreover the solution operator $\mathcal{R}[\mathbf{v}, \lambda]$ of problem $\mathcal{P}[\mathbf{v}, \lambda]$ is meromorphic in $\lambda \in \mathbb{C}$, the set of its poles is $\mathcal{S}[\mathbf{v}]$ and its polar part in any $\lambda \in \mathcal{S}[\mathbf{v}]$ has a finite range.

\subsection{Edges}

Let $\mathbf{e}$ be a point in an edge $E$. We assume that there exists a local chart which transforms in a neighborhood of $\mathbf{e}, \Omega$ and the $\Omega_{j}$ having $\mathbf{e}$ in their boundaries into wedges $\mathbb{R} \times \Gamma_{\mathbf{e}}$ and $\mathbb{R} \times \Gamma_{\mathbf{e}, j}$. By this local chart, problem (2) is transformed into a similar problem in the wedge $\mathbb{R} \times \Gamma_{\mathrm{e}}$. In cylindrical coordinates $(r, \theta, z)$, with $z \in \mathbb{R}$ and $(r, \theta)$ polar coordinates in the sector $\Gamma_{\mathrm{e}}$, the operators $L, B$ and $N$ can be formally expanded in increasing powers of $r$ according to:

$$
\left\{\begin{array}{l}
L=r^{-2} \mathcal{L}\left(\theta ; r \partial_{r}, \partial_{\theta}\right)+\sum_{p \geq 1} r^{-2+p} \mathcal{L}_{p}\left(\theta ; r \partial_{r}, \partial_{\theta}, \partial_{z}\right) \\
B=r^{-\operatorname{deg} B} \mathcal{B}\left(\theta ; r \partial_{r}, \partial_{\theta}\right)+\sum_{p \geq 1} r^{-\operatorname{deg} B+p} \mathcal{B}_{p}\left(\theta ; r \partial_{r}, \partial_{\theta}, \partial_{z}\right) \\
N=r^{-1} \mathcal{N}\left(\theta ; r \partial_{r}, \partial_{\theta}\right)+\sum_{p \geq 1} r^{-1+p} \mathcal{N}_{p}\left(\theta ; r \partial_{r}, \partial_{\theta}, \partial_{z}\right)
\end{array}\right.
$$

To the operators $\mathcal{L}, \mathcal{B}$ and $\mathcal{N}$ are associated their Mellin symbols in $r$, similarly to (5): $r \partial_{r}$ is changed into $\lambda$. The important point is that the tangential derivative $\partial_{z}$ along the edge does not appear in these symbols. With $G_{\mathbf{e}}$ and $G_{\mathbf{e}, j}$ the angular intervals corresponding to $\Gamma_{\mathbf{e}}$ and $\Gamma_{\mathbf{e}, j}$, we can define the operator valued Mellin symbol (which is now an ordinary 
differential operator)

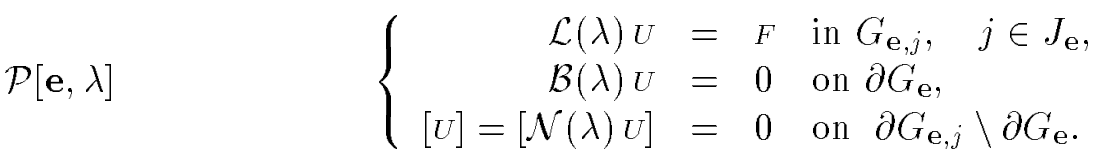

And similarly to the vertices, there holds

Lemma 4.2 The solution operator $\mathcal{R}[\mathrm{e}, \lambda]$ of problem $\mathcal{P}[\mathrm{e}, \lambda]$ is meromorphic, the set of its poles is a discrete subset $\mathcal{S}[\mathbf{e}]$ of $\mathbb{C}$ and its polar part in any $\lambda \in \mathcal{S}[\mathbf{e}]$ has a finite range. The intersection of the set $\mathcal{S}[\mathbf{e}]$ with any strip of the form $\operatorname{Re} \lambda \in[a, b]$ is finite.

\section{$5 \quad$ Singularities and regularity at conical points}

In order to explain the basic ideas which lead to regularity and expansion results, let us, only in this section, consider the case where $\Omega \subset \mathbb{R}^{n}$ has only one singular point (vertex) in $\mathbf{v}=0$, in the neighborhood of which it coincides with a cone $\Gamma_{\mathbf{v}}$ with (smooth) solid angle $G_{\mathbf{v}}$. We assume moreover that $L$ is homogeneous with constant coefficients and $B$ is the Dirichlet operator. Let us consider $u \in H_{0}^{1}(\Omega)$ such that $L u=f \in H^{s-1}(\Omega)$.

By a cut-off procedure, we can restrict ourselves to the case when $u$ in $H_{0}^{1}\left(\Gamma_{\mathbf{v}}\right)$ with compact support is such that $L u \in H^{s-1}\left(\Gamma_{\mathbf{v}}\right)$. Applying the Mellin transform to the equation $\rho^{2} L u=\rho^{2} f=: g$, we obtain

$$
\mathcal{R}[\mathbf{v}, \lambda](\mathcal{M}[g](\lambda))=\mathcal{M}[u](\lambda), \quad \forall \lambda, \operatorname{Re} \lambda \leq 1-\frac{n}{2} .
$$

Both sides are analytic functions for $\operatorname{Re} \lambda<1-\frac{n}{2}$. Since, because of the regularity of $f$, the Mellin transform of $g$ is defined (as a meromorphic function) for $\operatorname{Re} \lambda \leq s+1-\frac{n}{2}$, formula (7) yields a meromorphic extension $U$ of $\mathcal{M}[u]$ for $\operatorname{Re} \lambda \in\left[1-\frac{n}{2}, s+1-\frac{n}{2}\right]$.

If $U$ has no pole on the straight line $\operatorname{Re} \lambda=s+1-\frac{n}{2}$, let $u_{0}$ be the inverse Mellin transform of $U$ on this line:

$$
u_{0}(\rho)=\frac{1}{2 \pi} \int_{-\infty}^{+\infty} \rho^{\xi+i \eta} U(\xi+i \eta) d \eta \quad \text { with } \quad \xi=s+1-\frac{n}{2} .
$$

An extension of the residue formula to the unbounded contour $\operatorname{Re} \lambda=1-\frac{n}{2}$ and $\operatorname{Re} \lambda=$ $s+1-\frac{n}{2}$ yields, see KONDRAT'EV [24]:

$$
u_{0}-u=\sum_{1-\frac{n}{2}<\operatorname{Re} \lambda_{0}<s+1-\frac{n}{2}} \operatorname{Res}_{\lambda=\lambda_{0}} \rho^{\lambda} \mathcal{R}[\mathbf{v}, \lambda](\mathcal{M}[g](\lambda)) .
$$

Note that the proof of this formula relies on a priori elliptic estimates for the operator $L$ with its boundary conditions in a smooth part of the cone $\Gamma_{\mathbf{v}}$ (away from the corner).

If $\chi$ is a smooth cut-off function which is equal to 1 in a neighborhood of 0 and equal to 0 away from a larger neighborhood where $\Omega$ coincides with $\Gamma_{\mathbf{v}}$, as a consequence of (8)

$$
\chi u_{0} \in H^{s+1}(\Omega) .
$$


It remains to analyze the residues in formula (9). The $\lambda_{0}$ occurring in this formula are either poles of the solution operator $\mathcal{R}[\mathbf{v}, \lambda]\left(\lambda_{0} \in \mathcal{S}[\mathbf{v}]\right)$ or poles of the Mellin transform of the right hand side $\mathcal{M}[g](\lambda)$ : this latter poles are located on the only integers $d \geq 2$ and the corresponding residues are the Taylor part (homogeneous of degree $d$ ) of $g$ in 0 . As a consequence of Lemma 4.1, the right hand side of (9) involves a finite set in $\mathbb{C}$ $\left(\lambda_{0} \in \mathcal{S}[\mathbf{v}] \cup \mathbb{N}_{2}\right.$, with $\mathbb{N}_{2}$ the set of integers $\geq 2$ ). Moreover, for each $\lambda_{0} \in \mathcal{S}[\mathbf{v}] \cup \mathbb{N}_{2}$, the corresponding residue lives in a finite dimension space. We have to investigate the nature of its elements.

The residues in $\lambda_{0}$ have the general form

$$
\sum_{q=0}^{Q} \rho^{\lambda_{0}} \log ^{q} \rho U_{q}(\vartheta)
$$

with, in the particular situation of this section, $U_{q} \in H_{0}^{1}\left(G_{\mathbf{v}}\right) \cap \mathcal{C}^{\infty}\left(\bar{G}_{\mathbf{v}}\right)$.

If $\lambda_{0} \notin \mathbb{N}$, as $\operatorname{Re} \lambda_{0}<s+1-\frac{n}{2}$, any non-zero term $u_{\mathbf{v}, \lambda_{0}}$ (11) satisfies $\chi u_{\mathbf{v}, \lambda_{0}} \notin$ $H^{s+1}(\Omega)$ (although satisfying $L\left(\chi u_{\mathbf{v}, \lambda_{0}}\right) \in \mathcal{C}^{\infty}(\bar{\Omega})$ and the boundary conditions). All these terms are singular functions and contribute to the singular part.

If $\lambda_{0} \in \mathbb{N}$, some of the residues can be homogeneous polynomials (in cartesian variables of course) of degree $\lambda_{0}$ and do not contribute to the singular part.

That is the reason for the introduction of the following definitions.

\section{Definition 5.1}

i) For any $\lambda_{0} \in \mathcal{S}[\mathbf{v}] \cup \mathbb{N}_{2}$, we denote by

$$
u_{\mathbf{v}, \lambda_{0}}^{k}, \quad \text { for } k=1, \ldots, K_{\mathbf{v}, \lambda_{0}}
$$

a basis of non-polynomial residues $\operatorname{Res}_{\lambda=\lambda_{0}} \rho^{\lambda} \mathcal{R}[\mathbf{v}, \lambda] F(\lambda)$ when $F$ spans meromorphic functions with poles at integers and corresponding Taylor polar part, with the convention that $K_{\mathbf{v}, \lambda_{0}}=0$ if all residues are polynomials.

ii) We denote by $\widetilde{\mathcal{S}}[\mathbf{v}]$ the subset of $\lambda_{0} \in \mathcal{S}[\mathbf{v}] \cup \mathbb{N}_{2}$ such that $K_{\mathbf{v}, \lambda_{0}} \neq 0$.

iii) We denote by $\xi_{\mathbf{v}}$ the least real part $>1-\frac{n}{2}$ of $\lambda_{0} \in \widetilde{\mathcal{S}}[\mathbf{v}]$.

Thus, $\xi_{\mathbf{v}}$ is the least real part of active singular functions. The main result is then

Theorem 5.2 Let $u \in H_{0}^{1}(\Omega)$ be such that $L u=f \in H^{s-1}(\Omega)$. If the straight line $\operatorname{Re} \lambda=s+1-\frac{n}{2}$ does not meet the pseudo-spectrum $\widetilde{\mathcal{S}}[\mathbf{v}]$, there holds:

a) If $s<\xi_{\mathbf{v}}$, then $u \in H^{s+1}(\Omega)$.

b) If $s>\xi_{\mathbf{v}}$, then $u \in H^{\sigma+1}(\Omega)$ for all $\sigma<\xi_{\mathbf{v}}$ and there exist coefficients $\gamma_{\mathbf{v}, \lambda}^{k}$ such that

$$
u-\sum_{\substack{\lambda \in \tilde{\mathcal{S}}[\mathbf{v}], 1-\frac{n}{2}<\operatorname{Re} \lambda<s+1-\frac{n}{2}}} \sum_{k=1}^{K_{\mathbf{v}, \lambda}} \gamma_{\mathbf{v}, \lambda}^{k} \chi u_{\mathbf{v}, \lambda}^{k} \in H^{s+1}(\Omega) .
$$


With the strongest assumption "the straight line $\operatorname{Re} \lambda_{0}=s+1-\frac{n}{2}$ does not meet $\mathcal{S}[\mathbf{v}] \cup \mathbb{N}_{2}$ ", the previous statement is not too difficult to prove. But the assumption of the theorem hides serious difficulties, which have to be overcome since this assumption is exactly the right one (necessary and sufficient condition to have the result) and allows to recover integer Sobolev exponents in general in dimension $n=2$, see [16].

Definition 5.1 seems very complicate, due to the possible interaction between polynomials and poles of the solution operator $\mathcal{R}[\mathbf{v}, \lambda]$ of the operator valued symbol $\mathcal{P}[\mathbf{v}, \lambda]$. But, in a generic way, the situation is much simpler. We have first to explain what we mean by generic.

The sets $\mathcal{S}[\mathbf{v}]$ or $\widetilde{\mathcal{S}}[\mathbf{v}]$ depend on $L$, the nature of boundary conditions and the shape of $G_{\mathbf{v}}$. Indeed, they depend continuously on a family of parameters which are living in a finite (if $n=2$ ) or infinite dimensional domain. We will say that a situation is generic if it occurs only in a closed subset of measure zero in the parameter domain. In this sense:

Remark 5.3 In a generic way there holds

1. If $\lambda_{0} \in \mathcal{S}[\mathbf{v}]$ and $\lambda_{0} \notin \mathbb{N}$, the pole of $\mathcal{R}[\mathbf{v}, \lambda]$ in $\lambda_{0}$ is of order 1 and the polar part is an operator of rank 1 , whose range is generated by a basis $U_{\mathbf{v}, \lambda_{0}}$ of the kernel of $\mathcal{P}\left[\mathbf{v}, \lambda_{0}\right]$. The associated singularity space is also of dimension 1 , generated by $u_{\mathbf{v}, \lambda_{0}}=\rho^{\lambda_{0}} U_{\mathbf{v}, \lambda_{0}}(\vartheta)$.

2. If $n=2, \mathcal{S}[\mathbf{v}]=\widetilde{\mathcal{S}}[\mathbf{v}]$ (this fails only when the opening of $G_{\mathbf{v}}$ is $2 \pi$, i.e. if $\Omega$ has a (rack).

3. If $n \geq 3, \widetilde{\mathcal{S}}[\mathbf{v}]=\mathcal{S}[\mathbf{v}] \cup \mathbb{N}_{2}$, and for $\lambda_{0} \in \mathbb{N}_{2}$ the associated singular function space has the same dimension as the space of homogeneous polynomials of degree $\lambda_{0}-2$ and all its elements have the form $\rho^{\lambda_{0}} U(\vartheta)$.

\section{Regularity results}

Let us go back to the situation of section 4 . From the solution operators $\mathcal{R}[\mathbf{v}, \lambda]$ and $\mathcal{R}[\mathbf{e}, \lambda]$ we define the pseudo-spectra $\widetilde{\mathcal{S}}[\mathbf{v}]$ and $\widetilde{\mathcal{S}}[\mathbf{e}]$ exactly as in Definition 5.1 and we denote

$$
\left\{\begin{array}{l}
\xi_{\mathbf{v}} \text { the least real part }>-\frac{1}{2} \text { of } \lambda_{0} \in \widetilde{\mathcal{S}}[\mathbf{v}], \\
\xi_{\text {e }} \text { the least real part }>0 \text { of } \lambda_{0} \in \widetilde{\mathcal{S}}[\mathbf{e}] .
\end{array}\right.
$$

Then there holds

Theorem 6.1 If for all vertices $\mathbf{v}, s<\xi_{\mathbf{v}}+\frac{1}{2}$ and for all points $\mathrm{e}$ in any closed edge $E$, $s<\xi_{\mathrm{e}}$, then there holds the following regularity result: if $f \in P H^{s-1}(\Omega)$, see (3), then the solution $u$ of problem (1) belongs to $\mathrm{PH}^{s+1}(\Omega)$.

We refer to Maz'Ya, Plamenevskit $[35,34]$ for sharp regularity results in $L^{p}$ Sobolev spaces. 
This statement can be extended to any three-dimensional domain (possibly decomposed into sub-domains) having edges in the sense of section 4 and also vertices which are conical points. The requirement is that there exists a smooth local map which transforms a neighborhood of such a vertex into homogeneous cone(s) and that the transformed operators can be expanded like in (4). In the situation of Figure 4 (an ordinary cone, a hollow
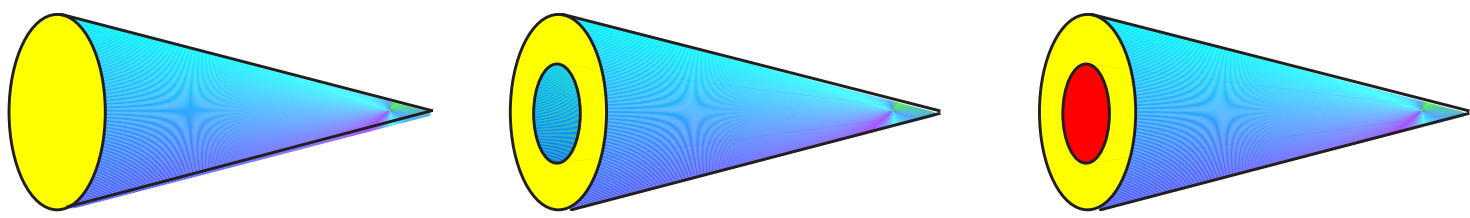

Figure 4: Conical points

cone or two materials filling two nested cones), Dirichlet or Neumann conditions associated with a general integro-differential form as in section 2 satisfy the required conditions.

Thus it can be important to have a lower bound for $\xi_{\mathbf{v}}$ and $\xi_{\mathbf{e}}$. For $L=-\Delta, \xi_{\mathbf{v}}$ is always larger than 0 , see [16, Ch. 19] for instance. For Dirichlet boundary conditions and $L$ the Lamé system or the Stokes system, we have $\xi_{\mathrm{v}}>0$ also, see KozLov, MAz'YA, Schwa $[28,29]$, and for mixed boundary conditions, see Kozlov, MaZ'Ya, Rossmann $[27,26]$.

Concerning $\xi_{\mathrm{e}}$, for $L=-\Delta$ with Dirichlet or Neumann boundary conditions, we have $\xi_{\mathrm{e}}>\frac{1}{2}$ for any Lipschitz sector, and $\xi_{\mathbf{e}}=\frac{1}{2}$ if $\Gamma_{\mathbf{e}}$ is the sector of opening $2 \pi$. Combining this with the previous estimates on $\xi_{\mathbf{v}}$ we obtain that for a Lipschitz polyhedron the upper bound on $s$ for the regularity result to hold is $>\frac{1}{2}$, compare with the Lipschitz theory Jerison, Kenig $[22,23]$. For Lamé and Stokes systems with Dirichlet conditions, we still have $\xi_{\mathrm{e}} \geq \frac{1}{2}$.

But the situation can be very different when there are transmission conditions. Let us give the example of the equation of the electrostatic potential associated with the form $a(u, v)=\sum_{j} \int_{\Omega_{j}} \varepsilon_{j} \nabla u \cdot \nabla v d x$, with $\varepsilon_{j}>0$ and exterior Dirichlet conditions. We give in the following Table 1 the optimal minima for $\xi_{\mathrm{e}}$ according to the number of materials joining at $\mathbf{e}$ and according to the opening of the full sector $\Gamma_{\mathbf{e}}$ (exterior angle), see [15] for the proofs. The mention "none" means that the cone $\Gamma_{\mathbf{e}}$ fills the whole space $\mathbb{R}^{2}$.

\begin{tabular}{|l|c|c|c|c|}
\hline Exterior Angle & 1 material & 2 materials & 3 materials & 4 materials \\
\hline$\leq \frac{\pi}{2}$ & 2 & 1 & 0 & 0 \\
Convex & 1 & $\frac{1}{2}$ & 0 & 0 \\
Any & $\frac{1}{2}$ & $\frac{1}{4}$ & 0 & 0 \\
None & $\infty$ & $\frac{1}{2}$ & $\frac{1}{4}$ & 0 \\
\hline
\end{tabular}

Table 1 : Optimal minima 
In Figure 5 we indicate the situations which produce the lower bound: two different values for the $\varepsilon_{j}$ (materialized by the two colors in the drawings) are enough but the ratio between these two values has to be very large or very small.
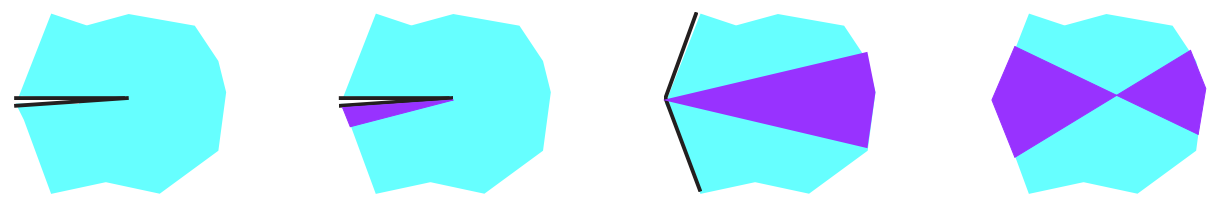

Figure 5: Limit situations for 1, 2, 3 or 4 materials

\section{Singularities}

We already gave in Theorem 5.2 the expansion into regular and singular parts at a conical point. Let us go back to the framework of section 4. At vertices $\mathbf{v}$ the singular functions

$$
(\rho, \vartheta) \longmapsto u_{\mathbf{v}, \lambda}^{k}
$$

are defined in the sense of Definition 5.1 (with $n=3$ ) and at any edge point $\mathbf{e}$, for any $\mu$ belonging to the pseudo-spectrum $\widetilde{\mathcal{S}}[\mathbf{e}]$ of the operator valued Mellin symbol $\mathcal{P}[\mathbf{e}, \cdot]$, the singular functions

$$
(r, \theta) \longmapsto u_{\mathbf{e}, \mu}^{k}
$$

are also defined in the sense of Definition 5.1 (with $n=2$ ).

\subsection{Straight polyhedra and constant coefficients}

In this subsection, we will present (in a simplified framework) the results of [16] concerning the expansion into regular and singular parts in a polyhedron. So, we assume

$$
\left\{\begin{array}{l}
\text { i) The domains } \Omega \text { and } \Omega_{j} \text { are polyhedra (i.e. with plane faces), } \\
\text { ii) The form } a \text { has piecewise constant coefficients (constant in each } \Omega_{j} \text { ), } \\
\text { iii) The singular functions } u_{\mathbf{v}, \lambda}^{k} \text { and } u_{\mathbf{e}, \lambda}^{k} \text { have no logarithmic term. }
\end{array}\right.
$$

As a consequence of the points $i$ ) and ii) of the assumption (14), neither the pseudo spectrum $\widetilde{\mathcal{S}}[\mathbf{e}]$ nor the singular functions $u_{\mathbf{e}, \mu}^{k}$ depend on the fixed point $\mathbf{e} \in E$ : they only depend on $E$ and can be denoted $\widetilde{\mathcal{S}}[E]$ and $u_{E, \mu}^{k}$ respectively. From the expansion (12) we can guess that to each vertex and each edge will be associated a part of the expansion and that the edge parts will involve coefficients along the edges.

In order to give a precise statement, we need weighted Sobolev spaces for these edge singularity coefficients and a smoothing operator. Let us fix an edge $E$ : we recall that $(r, \theta, z)$ are cylindrical coordinates associated to this edge and let

$$
\delta: \bar{E} \ni z \longmapsto \delta(z) \in \mathbb{R}
$$


be a smooth function on $\bar{E}$, equivalent to the distance to the end points of $E$ : if for example $E=\{x \mid r=0, z \in(-1,+1)\}$, we can take $\delta(z)=1-z^{2}$.

For $m \in \mathbb{N}$ and $\eta \in \mathbb{R}$, let $V_{\eta}^{m}(E)$ be the weighted Sobolev space defined as

$$
V_{\eta}^{m}(E)=\left\{\gamma \in L^{2}(E) \mid \quad \delta^{\eta+k} \partial_{z}^{k} \gamma \in L^{2}(E), \quad k=0,1, \ldots, m\right\}
$$

and by interpolation for non-integer $m$.

The smoothing operator $\mathcal{K}[\cdot]$ acts like a lifting of functions on $E$ into $\Omega$ : in order to define it, we need two different stretched variables. Firstly

$$
\tilde{r}=\frac{r}{\delta}
$$

We call the triple $(\tilde{r}, \theta, z)$ the stretched cylindrical coordinates. The inequalities $\tilde{r}<\tilde{r}_{0}$ define a sort of sectorial neighborhood of $E$.

And secondly

$$
\tilde{z}=\int_{z_{0}}^{z} \frac{1}{\delta(\zeta)} d \zeta
$$

where $z_{0}$ corresponds to an interior point of $E$. The change of variable $z \mapsto z$ is one to one $E \rightarrow \mathbb{R}$ and for any function $\gamma$ defined on $E$, we set $\tilde{\gamma}(\tilde{z})=\gamma(z)$.

Then $\mathcal{K}[\gamma](\tilde{r}, z)$ is defined as the convolution operator with respect to $\tilde{z}$ :

$$
\mathcal{K}[\gamma](\tilde{r}, z)=\int_{\mathbb{R}} \frac{1}{\tilde{r}} \varphi\left(\frac{t}{\tilde{r}}\right) \tilde{\gamma}(t-\tilde{z}) d t
$$

where $\varphi$ is a smooth function in $\mathcal{S}(\mathbb{R})$ such that $\int_{\mathbb{R}} \varphi=1$. Thus for any fixed $\tilde{r}_{0} \neq 0$, the function $z \mapsto \mathcal{K}[\gamma]\left(\tilde{r}_{0}, z\right)$ is smooth inside $E$, and tends to $\gamma$ as $\tilde{r}_{0} \rightarrow 0$.

Finally, we denote, with a smooth cut-off $\chi$ equal to 1 in a neighborhood of 0 and with small enough support

$$
U_{\mathbf{v}, \lambda}^{k}(x)=\chi(\rho) u_{\mathbf{v}, \lambda}^{k}(\rho, \vartheta) \quad \text { and } \quad U_{E, \mu}^{k}(x)=\chi(\tilde{r}) u_{E, \mu}^{k}(\tilde{r}, \theta) .
$$

It is important to note that there exists $\tilde{r}_{0}>0$ such that for all $\tilde{r} \leq \tilde{r}_{0}$ and all $\theta \in G_{E}$, the point of stretched cylindrical coordinates $(\tilde{r}, \theta, z)$ belongs to $\Omega$.

Theorem 7.1 We assume (14). Let $u$ be the solution of problem (1) with $f \in P H^{s-1}(\Omega)$. If for any vertex $\mathbf{v}$ the straight line $\operatorname{Re} \lambda=s-\frac{1}{2}$ does not meet the pseudo-spectrum $\widetilde{\mathcal{S}}[\mathbf{v}]$, and if for any edge $E$ the straight line $\operatorname{Re} \mu=s$ does not meet the pseudo-spectrum $\widetilde{\mathcal{S}}[E]$, there holds with

$$
s_{0}=\min \left\{\min _{\mathbf{v} \text { vertex }} \xi_{\mathbf{v}}+\frac{1}{2}, \min _{\mathbf{e} \in \text { edge }} \xi_{\mathbf{e}}\right\}:
$$

a) If $s<s_{0}$, then $u \in P H^{s+1}(\Omega)$ (Theorem 6.1 ).

b) If $s_{0} \leq s<s_{0}+1$, then $u \in H^{\sigma+1}(\Omega)$ for all $\sigma<s_{0}$ and there exist coefficients $\gamma_{\mathbf{v}, \lambda}^{k} \in \mathbb{C}$ and functions $\gamma_{E, \mu}^{k} \in V_{-s}^{s-\operatorname{Re} \mu}(E)$ such that 


$$
\begin{aligned}
& u-\sum_{\mathbf{v} \text { vertex }} \sum_{\substack{\lambda \in \tilde{\mathcal{S}}[\mathbf{v}],-\frac{1}{2}<\operatorname{Re} \lambda<s-\frac{1}{2}}} \sum_{k=1}^{K_{\mathbf{v}, \lambda}} \gamma_{\mathbf{v}, \lambda}^{k} U_{\mathbf{v}, \lambda}^{k} \\
&-\sum_{E \text { edge }} \sum_{\substack{\mu \in \widetilde{\mathcal{S}}[E], 0<\operatorname{Re} \mu<s}} \sum_{k=1}^{K_{E, \mu}} \mathcal{K}\left[\gamma_{E, \mu}^{k}\right] U_{E, \mu}^{k} \in H^{s+1}(\Omega) .
\end{aligned}
$$

c) If $s \geq s_{0}+1, u$ still admits an expansion similar to (18), but with new sorts of terms, the shadow terms:

$$
\gamma_{\mathbf{v}, \lambda}^{k} U_{\mathbf{v}, \lambda}^{k, p} \quad \text { and } \quad \mathcal{K}\left[\partial_{z}^{p} \gamma_{E, \mu}^{k}\right] U_{E, \mu}^{k, p}, \quad p=1,2, \ldots
$$

The degree of homogeneity of the functions $U_{\mathbf{v}, \lambda}^{k, p}$ and $U_{E, \mu}^{k, p}$ is $\lambda+p$ and $\mu+p$ respectively.

For a complete statement in the situation satisfying to $i$ ) and ii) of the assumption (14), but without excluding logarithmic singularities, see [16].

\subsection{Vertex - Edge interaction}

We still assume (14), and only in order to simplify the notations, that the "multiplicities" $K_{\mathbf{v}, \lambda}$ and $K_{E, \mu}$ are equal to 1 , which allows to drop the exponent $k$ for the singular functions. We moreover assume that we are in situation $b$ ) of Theorem 7.1.

In Figure 6, the edge $E$ has the vertices $\mathbf{v} 1$ and $\mathbf{v} 2$ as end points. The vertices $\mathbf{v} 1$ and $\mathbf{v} 2$ correspond to spherical polygons $G 1$ and $G 2$ respectively. The corners of $G 1$ correspond to the edges $E, E 11$ and $E 12$ and the corners of $G 2$ correspond to the edges $E$, E21 and E22.
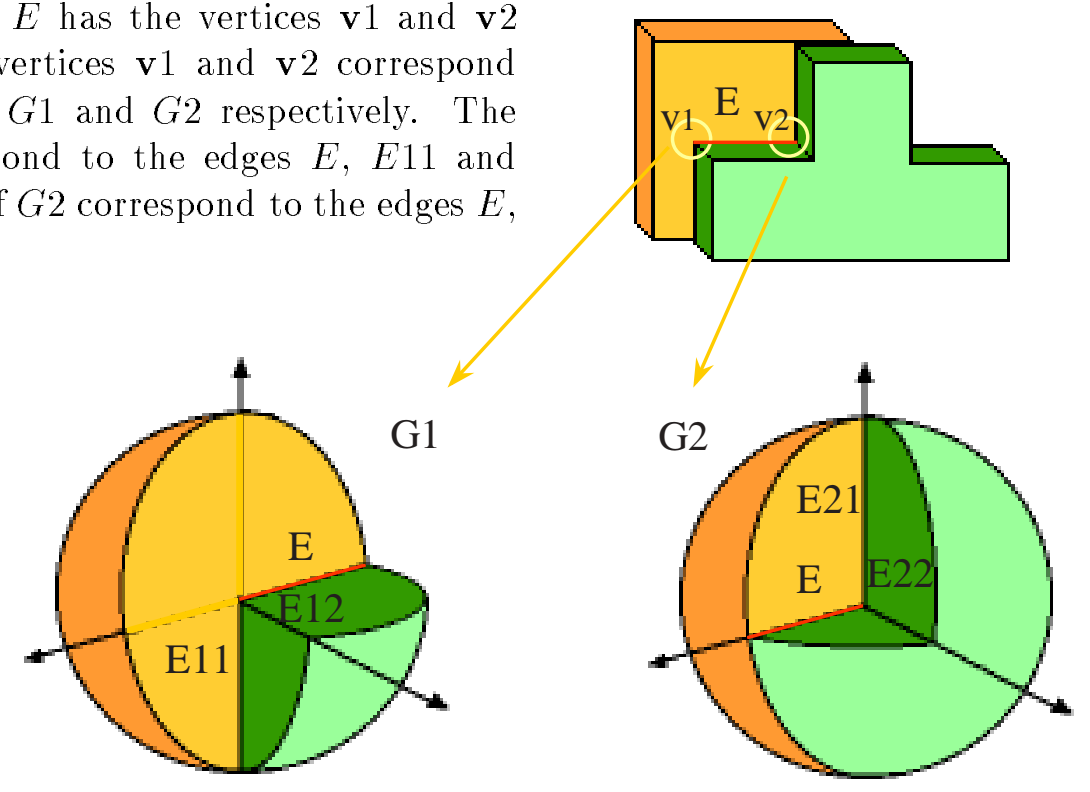

Figure 6: Vertices and edges 
The expansion (18) is obtained by first splitting the solution near the vertices and second along the edges. Splitting only along the edges yields new edge coefficients $\tilde{\gamma}_{E, \mu} \in$ $V_{0}^{s-\operatorname{Re} \mu}(E)$. It is interesting to compare $\gamma_{E, \mu}$ and $\tilde{\gamma}_{E, \mu}$.

The vertex singular functions are $\rho^{\lambda} U_{\mathbf{v}, \lambda}(\vartheta)$ and each $U_{\mathbf{v}, \lambda}$ has singularities at the corners of $G$ (corresponding to the edges such that $\bar{E} \ni \mathbf{v}$ ): these singularities are the already introduced $U_{E, \mu}$ and there exist coefficients $a_{E, \mu}^{\mathbf{v}, \lambda}$ such that there holds

$$
U_{\mathbf{v}, \lambda}-\sum_{\bar{E} \ni \mathbf{v}} \sum_{\substack{\mu \in \widetilde{\mathcal{S}}[E] \\ 0<\operatorname{Re} \mu<s}} a_{E, \mu}^{\mathbf{v}, \lambda} U_{E, \mu} \in H^{s+1}(G) .
$$

By plugging this expansion into splitting (18) we obtain the following expansion of the edge coefficients in a neighborhood of $\mathbf{v}$ :

$$
\tilde{\gamma}_{\mathbf{e}, \mu}-\sum_{\substack{\lambda \in \widetilde{\mathcal{S}}[\mathbf{v}] \\-1 / 2<\operatorname{Re} \lambda<s-1 / 2}} a_{E, \mu}^{\mathbf{v}, \lambda} \rho^{\lambda}=\gamma_{\mathbf{e}, \mu} .
$$

\subsection{Curved edges or variable coefficients}

In presence of curved edges or variable coefficients, the operator valued Mellin symbol $\mathcal{P}[\mathbf{e}, \cdot]$ and the singular functions $u_{\mathbf{e}, \mu}^{k}$ depend on $\mathbf{e} \in E$. Let us assume now that there are only edges and no vertices (since the edges are curved, this is possible!). A difficulty arises now, coming from possible changes in the multiplicity.

If we avoid this difficulty by the assumption that for all $\mathbf{e} \in E$ the algebraic and geometric multiplicities of the poles of the resolvent $\mathcal{R}[\mathbf{e}, \cdot]$ do not change in the strip $0<\operatorname{Re} \mu \leq s$ and that they never coincide with integers (in other words, there exists a way to define the bases $u_{\mathbf{e}, \mu}^{k}$ with a $\mathcal{C}^{\infty}$ dependence on $\mathbf{e} \in E$ ), then an expansion like

$$
u-\sum_{E \text { edge }} \sum_{\substack{\mu \in \widetilde{\mathcal{S}}[\mathbf{e}], 0<\operatorname{Re} \mu<s}} \sum_{k=1}^{K_{E, \mu}} \mathcal{K}\left[\gamma_{E, \mu}^{k}\right] U_{\mathbf{e}, \mu}^{k} \in H^{s+1}(\Omega)
$$

holds with coefficients which belong to Sobolev spaces with variable exponents, see MAZ'YA, Rossmann [36] and Nazarov, Plamenevskit [39]: if $\mu$ is a simple pole $\gamma_{E, \mu}^{k} \in H^{s-\operatorname{Re} \mu}(E)$.

But the above assumption is not generic. Two basic situations escape to this assumption

i) For $\mathbf{e}_{0} \in E$ and $\mu_{0} \in \mathcal{S}\left[\mathbf{e}_{0}\right]$, the resolvent $\mu \longmapsto \mathcal{R}\left[\mathbf{e}_{0}, \mu\right]$ has a double pole and for

$\mathbf{e} \in E, \mathbf{e} \neq \mathbf{e}_{0}$ there are two simple poles $\mu_{1}(\mathbf{e}) \rightarrow \mu_{0}$ and $\mu_{2}(\mathbf{e}) \rightarrow \mu_{0}$ as $\mathbf{e} \rightarrow \mathbf{e}_{0}$.

This is the classical situation of a branching ${ }^{1}$. In any $\mathbf{e} \in E$ near $\mathbf{e}_{0}$, there exist two

\footnotetext{
${ }^{1}$ The well known example is the first singular function of the Stokes Dirichlet problem: $\mathbf{e}_{0}$ corresponds to the opening $\omega=0.813 \pi$ and $\mathbf{e} \neq \mathbf{e}_{0}$ to different openings.
} 
associated singularities: in $\mathbf{e}_{0}$ these are

$$
r^{\mu_{0}} U_{\mathbf{e}_{0}, \mu_{0}}(\theta) \quad \text { and } \quad r^{\mu_{0}}\left(\log r U_{\mathbf{e}_{0}, \mu_{0}}(\theta)+V \mathbf{e}_{0}, \mu_{0}(\theta)\right)
$$

and for $\mathbf{e} \neq \mathbf{e}_{0}$, these are

$$
u_{1}(\mathbf{e}):=r^{\mu_{1}} U_{\mathbf{e}, \mu_{1}}(\theta) \quad \text { and } \quad u_{2}(\mathbf{e}):=r^{\mu_{2}} U_{\mathbf{e}, \mu_{2}}(\theta) .
$$

ii) The pole $\mu$ is simple and depends smoothly on $\mathbf{e} \in E$ but for an isolated value $\mathbf{e}_{0} \in E$, $\mu=\mu_{0} \in \mathbb{N}_{2}$ and interacts with polynomials. This is the case of the crossing ${ }^{2}$. There is only one singular function for each value of $\mathbf{e}$ but it has different expressions if $\mathrm{e}=\mathbf{e}_{0}$

$$
r^{\mu_{0}}\left(\log r U_{\mathbf{e}_{0}, \mu_{0}}(\theta)+V_{\mathbf{e}_{0}, \mu_{0}}(\theta)\right)
$$

and if $\mathbf{e} \neq \mathbf{e}_{0}$

$$
r^{\mu} U_{\mathbf{e}, \mu}
$$

In this situation $r^{\mu_{0}} U_{\mathbf{e}_{0}, \mu_{0}}(\theta)$ is a polynomial in cartesian variables ${ }^{3}$ and is the limit of $r^{\mu} U_{\mathbf{e}, \mu}$ as $\mathbf{e} \rightarrow \mathbf{e}_{0}$. Then we set $\mu_{1}(\mathbf{e})=\mu(\mathbf{e})$ and $\mu_{2}(\mathbf{e}) \equiv \mu_{0}$, and

$$
u_{1}(\mathbf{e}):=r^{\mu_{1}} U_{\mathbf{e}, \mu_{1}} \quad \text { and } \quad u_{2}(\mathbf{e}):=r^{\mu_{0}} U_{\mathbf{e}_{0}, \mu_{0}} .
$$

In both situations, if we try to write an expansion of the solution $u$ with the original singular functions $u_{1}(\mathbf{e})$ and $u_{2}(\mathbf{e})$ in the case of branching, and $u_{1}(\mathbf{e})$ in the case of crossing respectively, we obtain coefficients which behave in general like $\left(\mu_{1}-\mu_{2}\right)^{-1}$ and thus blow up as $\mathbf{e} \rightarrow \mathbf{e}_{0}$. We obtain Stable Singularities by taking the sum and the divided difference of the previous expressions

$$
\widetilde{u}_{1}(\mathbf{e})=u_{1}(\mathbf{e})+u_{2}(\mathbf{e}) \quad \text { and } \quad \widetilde{u}_{2}(\mathbf{e})=\frac{u_{1}(\mathbf{e})-u_{2}(\mathbf{e})}{\mu_{1}(\mathbf{e})-\mu_{2}(\mathbf{e})} .
$$

These new singular functions depend smoothly on $\mathbf{e} \in E$ and yield asymptotic expansions like (19) with coefficients which do not blow up as $\mathbf{e} \rightarrow \mathbf{e}_{0}$, see Costabel, Dauge $[10,11]$. We note that in case ii), we have to combine a singular function with a polynomial (which is a regular function). Here the correct concept is that of asymptotic expansion rather that of splitting into regular and singular parts.

\section{Effects of singularities on finite element discretizations}

The first obvious effect of singularities is the limitation of the regularity of solutions, even if the data are very smooth. This fact hampers the convergence of Galerkin methods based on finite element discrete spaces for the discretization of problem (1).

\footnotetext{
${ }^{2}$ The well known example is the first singular function of the Laplace Dirichlet problem: $\mathbf{e}_{0}$ corresponds to the opening $\omega=\pi / 2$ and $\mathbf{e} \neq \mathbf{e}_{0}$ to different openings. Then $\mu_{0}=2$.

${ }^{3}$ For the previous example $U_{\mathbf{e}_{0}, \mu_{0}}(\theta)=\sin 2 \theta$ and $r^{\mu_{0}} U_{\mathbf{e}_{0}, \mu_{0}}(\theta)=2 x y$.
} 
Concerning the classical $h$-version of finite elements, if a regular and uniform mesh is used with continuous piecewise polynomials of degree $d$, then the maximal convergence rate in energy norm $H^{1}(\Omega)$ is, with $s_{0}$ the upper bound (17) for the regularity, see [6] for instance

$$
h^{\min \left\{d, s_{0}\right\}},
$$

where $h$ is the characteristic length of the mesh. As may be seen from Table $1, s_{0}$ can be very small.

Concerning the $p$-version of finite elements or the spectral element method, where a mesh is fixed and the degree $N$ of the polynomial spaces is increased, from the regularity of the solution $u$ of (1) in Sobolev spaces we can deduce that the convergence rate in energy norm is $N^{-s_{0}}$. But this result is not optimal, indeed, for smooth enough data, the convergence rate is doubled: it is

$$
N^{-2 s_{0}}
$$

see DorR [19] for the $p$-version and BERNARDi, MADAY [5] for the spectral method.

Let us give the main arguments leading to this improved convergence rate in the framework of spectral methods. Let $\Lambda$ be the model interval $(-1,1)$. The best approximation by polynomials of degree $N$ in $L^{2}(\Lambda)$ of a function $\psi$ behaves like $N^{-\sigma}$ not only if $\psi$ belongs to $H^{\sigma}(\Omega)$, but also if $\psi$ belongs to the domain of the operator $A^{\sigma / 2}$, where $A$ is the Sturm-Liouville operator on the interval $\Lambda$ :

$$
A \psi(\zeta)=\left(\left(1-\zeta^{2}\right) \psi^{\prime}\right)^{\prime}, \quad \zeta \in \Lambda
$$

whose eigenvalues are $N(N+1)$ and associated eigenfunctions are the Legendre polynomials of degree $N$. The domain of $A^{\sigma / 2}$ coincides with the weighted Sobolev space $H_{\sigma / 2}^{\sigma}(\Lambda)$ where for $m \in \mathbb{N}, H_{\eta}^{m}(\Lambda)$ is defined as

$$
H_{\eta}^{m}(\Lambda)=\left\{\psi \in L^{2}(\Lambda) \mid\left(1-\zeta^{2}\right)^{\eta} \partial_{\zeta}^{k} \psi \in L^{2}(\Lambda), \quad k=0,1, \ldots, m\right\}
$$

and by interpolation for non-integer $m$ (compare with (16), there is no shift of the weight with the order of derivatives). In a rectangle or a parallelepiped $\Omega^{\prime}$, we have similar approximation properties with weighted spaces obtained by tensorization from $H_{\sigma / 2}^{\sigma}(\Lambda)$. Then the key argument is the following one: for a singular function $u$ of the form (11) with $\lambda_{0} \in \mathbb{C}$ there holds (with $n$ the dimension of the space)

$$
u \in H^{s}\left(\Omega^{\prime}\right) \quad \Longleftrightarrow \quad \operatorname{Re} \lambda_{0}>s-\frac{n}{2} \Longleftrightarrow u \in H_{s}^{2 s}\left(\Omega^{\prime}\right)
$$

The doubled convergence rate is basically a consequence of this and of the classical Céa inequality.

Thus, augmenting the degree is a priori more efficient than increasing the number of subdomains in the mesh. However, within the $h$-version, a refinement of the mesh using special gradings can restore optimal convergence rates, see RAUGEL [44] for polygons and APEL, NiCAISE [2] for polyhedra. With a geometrical grading and an augmentation of degree (the $h-p$ version of finite elements), an exponential convergence rate can be obtained if all data are analytic, see BABUŠKA, SURI [3]. The cost is a more difficult implementation and an increased condition number. 


\section{Effects of singularities: the Maxwell bug}

Classical time harmonic Maxwell equations are given by

$$
\operatorname{curl} E-i \omega \mu H=0 \text { and } \operatorname{curl} H+i \omega \varepsilon E=J \text { in } \quad \Omega \text {. }
$$

Here $E$ is the electric part and $H$ the magnetic part of the electromagnetic field, whereas $\varepsilon$ and $\mu$ are the permittivity and the permeability of the medium. The right hand side $J$ is the current density. The exterior boundary conditions on $\partial \Omega$ are those of the perfect conductor ( $n$ denotes the unit outer normal on $\partial \Omega$ ):

$$
E \times n=0 \quad \text { and } \quad H \cdot n=0 \quad \text { on } \quad \partial \Omega .
$$

When $\Omega$ is polyhedral, or if the medium is heterogeneous with polyhedral homogeneous subdomains $\Omega_{j}$ where $\varepsilon$ and $\mu$ are constant, the electromagnetic field has different kinds of singularities which are thoroughly described in $[12,15]$. The main difference with what we described in the previous sections is the variational space: Equations (20) and (21) can be transformed in elliptic boundary value problems associated with the form

$$
(u, v) \longmapsto \int_{\Omega} \operatorname{curl} u \cdot \operatorname{curl} v+\operatorname{div} u \cdot \operatorname{div} v
$$

involving $E$ or $H$ alone, but the variational space for the electric part $E$ is defined as

$$
X_{N}(\Omega)=\left\{u \in L^{2}(\Omega)^{3}\left|\operatorname{curl} u \in L^{2}(\Omega)^{3}, \operatorname{div} \varepsilon u \in L^{2}(\Omega), \quad E \times n\right|_{\partial \Omega}=0\right\}
$$

and the corresponding space $X_{T}(\Omega)$ for the magnetic part is defined similarly.

The remarkable feature is that $X_{N}(\Omega)$ or $X_{T}(\Omega)$ are not contained in $H^{1}(\Omega)^{3}$ in general. Even if $\varepsilon$ and $\mu$ are constant (what we assume from now on), we have for any polyhedron with plane faces

$$
X_{N}(\Omega)=H_{N}(\Omega) \Longleftrightarrow \Omega \text { is convex }
$$

where $H_{N}(\Omega):=X_{N}(\Omega) \cap H^{1}(\Omega)^{3}$. If $\Omega$ is not convex the singularities in $X_{N}(\Omega)$ are the gradients of the singular functions of the Dirichlet Laplace operator on $\Omega$. Moreover, the closure in $X_{N}(\Omega)$ of smooth vector fields with zero tangential component is $H_{N}(\Omega)$. The form (22) is coercive on any of the spaces $X_{N}(\Omega)$ and $H_{N}(\Omega)$ and yields different solutions in general if the variational problem is posed in $X_{N}(\Omega)$ or $H_{N}(\Omega)$, see Costabel $[7,8]$ and $[15]$.

Consequently, if the form (22) is discretized with subspaces contained in $H_{N}(\Omega)$ (continuous piecewise affine vector fields for example), the discrete solution converges to the (wrong) solution in $H_{N}(\Omega)$. The manifestation of this "bug" on eigenvalue computation is showed up in [13] where computations in L-shaped polygons with nodal elements (via an elasticity code) are compared to the correct results (obtained via eigenvalues for the Laplace operator, which coincide with the Maxwell eigenvalues). 


\section{Conclusions}

Singularities are not there only to give trouble to mathematicians, to their theories and numerical methods. They are a part of the correct description of phenomena. For example, the singularities along a crack front, especially in continuum mechanics, are very important. First they are strong, behaving in $r^{1 / 2}$ for displacements, whence in $r^{-1 / 2}$ for stresses. Second, the associated coefficients $c_{\mathbf{v}, \lambda}$ are called stress intensity factors and intervene in different fracture criteria, see Nazarov, Polyakova [40] and the references therein.

Indeed, even if the domain has not real sharp corners, but only regions where the curvature of its boundary is very large, see $\Omega$ in the middle of Figure 7 , the behavior of its solutions are almost singular. In the example of Figure 7 , the limit domain with corner is represented in $(a)$. The solutions of coercive elliptic boundary value problems on $\Omega$ can be expanded with respect to the curvature parameter (for example, the curvature radius $\varepsilon)$ as $\varepsilon \rightarrow 0$ and involves solutions of problems on the domain (a) but also corner layer terms whose profiles are solutions of problems on the unbounded domain $(b)$, see MAZ'YA, Nazarov, Plamenevskit [33].

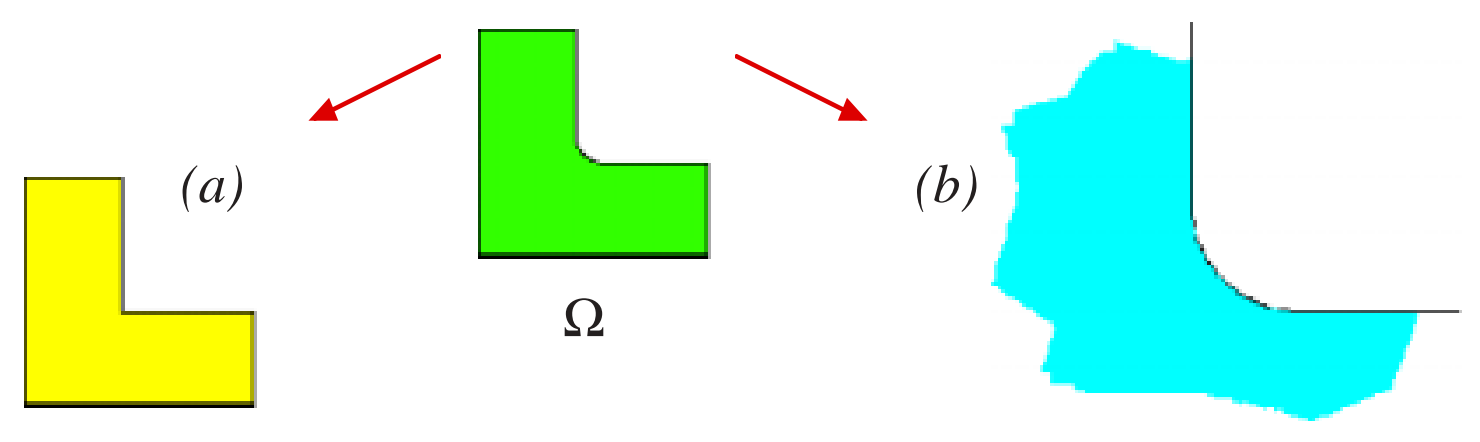

Figure 7: Curved corner

Thus, it can be important for theoretical or practical reasons, to know precisely the exponents $\lambda$ in the spectra $\mathcal{S}$ associated with $2 \mathrm{D}$ corners, edges or $3 \mathrm{D}$ vertices, and also the angular shape $U(\theta)$ or $U(\vartheta)$ of the singular functions. So let us end this short survey paper by a few references about the computation of singular functions.

For plane problems (associated with polygons or edges), concerning the Laplace operator and the Dirichlet or Neumann conditions the exponents are $k \pi / \omega$, for integer $k>0$, with $\omega$ the opening of the sector $\Gamma$. Concerning Lamé and Stokes operators, the spectrum coincides with the set of the zeros of special analytic functions, the root discriminant functions see Lozi [32] and Orlt, Sändig [43] for Stokes, Grisvard [21] and Nicaise, SÄNDIG [42] for isotropic elasticity. The determination of the root discriminant functions can be extended to any elliptic system in 2D, see Costabel, Dauge [9] and its implementation for general elasticity transmission problems in Costabel, Dauge, Lafranche [14]. Methods based on a more numerical approach are addressed in LEgUILLON [31] and 
Szabó, Yosibash $[47,48]$.

Very few works are devoted to the computation of three dimensional vertex singularities. Concerning the Laplace operator let us quote Stephan, Whiteman [46] and Beagles, Whiteman [4] using finite element approach on the solid angle $G$, or Schmitz, Volk, Wendland [45] using boundary element method on its boundary $\partial G$.

As a final conclusion, let us say that many regions are left to future mathematical or numerical explorations. The interaction of singularities with small (or large) parameters constitute one of these regions, endowed with very rich and various landscapes...

\section{References}

[1] S. Agmon, A. Douglis, L. Nirenberg. Estimates near the boundary for solutions of elliptic partial differential equations satisfying general boundary conditions II. Comm. Pure Appl. Math., 17, 1964, 35-92.

[2] T. Apel, S. Nicaise. The finite element method with anisotropic mesh grading for elliptic problems in domains with corners and edges. Math. Methods Appl. Sci, 21-6, 1998, 519-549.

[3] I. Babuška, M. Suri. The $p$ - and $h-p$ versions of the finite element method, an overview. Comput. Methods Appl. Mech. Engrg., 80-1\& 3, 1990, 5-26. Spectral and high order methods for partial differential equations (Como, 1989).

[4] A. E. Beagles, J. R. Whiteman. General conical singularities in three-dimensional Poisson problems. Math. Methods Appl. Sci, 11-2, 1989, 215-235.

[5] C. Bernardi, Y. Maday. Polynomial approximation of some singular functions. Appl. Anal., 42-1, 1991, 1-32.

[6] M. Bourlard, M. Dauge, M.-S. Lubuma, S. Nicaise. Coefficients of the singularities for elliptic boundary value problems on domains with conical points. III. Finite element methods on polygonal domains. SIAM J. Numer. Anal., 29-1, 1992, 136-155.

[7] M. Costabel. A remark on the regularity of solutions of Maxwell's equations on Lipschitz domains. Math. Methods Appl. Sci., 12-4, 1990, 365-368.

[8] M. Costa Bel. A coercive bilinear form for Maxwell's equations. J. Math. Anal. Appl., 157-2, $1991,527-541$.

[9] M. Costabel, M. Dauge. Construction of corner singularities for Agmon-Douglis-Nirenberg elliptic systems. Math. Nachr., 162, 1993, 209-237.

[10] M. Costabel, M. Dauge. General edge asymptotics of solutions of second order elliptic boundary value problems I \& II. Proc. Royal Soc. Edinburgh, 123-A, 1993, 109-184.

[11] M. Costabel, M. Dauge. Stable asymptotics for elliptic systems on plane domains with corners. Comm. Partial Differential Equations, 9 \& 10, 1994, 1677-1726.

[12] M. Costabel, M. Dauge. Singularities of electromagnetic fields in polyhedral domains. Preprint 97-19, Université de Rennes 1, 1997.

http://www.maths.univ-rennes1.fr/ dauge/.

ESAIM: PRoc., Vol. 6, SePtembre 1998, 19-40 
[13] M. Costabel, M. Dauge. Maxwell and Lamé eigenvalues on polyhedra. Preprint 98-08, Université de Rennes 1, 1998. To appear in Math. Meth. Appl. Sci. http://www.maths.univ-rennes1.fr/ dauge/.

[14] M. Costabel, M. Dauge, Y. Lafranche. Fast semi-analytic computation of elastic edge singularities. In preparation.

[15] M. Costabel, M. Dauge, S. Nicaise. Singularities of Maxwell interface problems. Preprint 98-24, Université de Rennes 1, 1998. To appear in Modél. Math. Anal. Numér. http://www.maths.univ-rennes1.fr/ dauge/.

[16] M. Dauge. Elliptic Boundary Value Problems in Corner Domains - Smoothness and Asymptotics of Solutions. Lecture Notes in Mathematics, 1341, Springer-Verlag, Berlin, 1988.

[17] M. Dauge. Strongly elliptic problems near cuspidal points and edges. In J. CEA, D. Chenais, G. Geymonat, J.-L. Lions, editors, Partial Differential Equations and Functional Analysis, In Memory of Pierre Grisvard, Progress in Nonlinear Differential Equations and Their Applications, Birkhäuser, Boston, 1996, 93-110.

[18] M. Dauge, S. Nicaise. Oblique derivative and interface problems on polygonal domains and networks. Comm. Partial Differential Equations, 14-8\& 9, 1989, 1147-1192.

[19] M. R. DorR. The approximation of solutions of elliptic boundary-value problems via the $p$-version of the finite element method. SIAM J. Numer. Anal, 23-1, 1986, 58-77.

[20] P. Grisvard. Boundary Value Problems in Non-Smooth Domains. Pitman, London, 1985.

[21] P. Grisvand. Singularités en élasticité. Arch. Rational Mech. Anal., 107-2, 1989, 157-180.

[22] D. S. Jerison, C. E. Kenig. The Neumann problem on Lipschitz domains. Bull. Amer. Math. Soc. (N.S.), 4-2, 1981, 203-207.

[23] D. S. Jerison, C. E. Kenig. Boundary value problems on Lipschitz domains. In Studies in partial differential equations, volume 23 of MAA Stud. Math., Math. Assoc. America, Washington D.C., 1982, 1-68.

[24] V. A. KondRat'Ev. Boundary-value problems for elliptic equations in domains with conical or angular points. Trans. Moscow Math. Soc., 16, 1967, 227-313.

[25] V. A. Kozlov. Coefficients in the asymptotics of the solutions of initial-boundary value parabolic problems in domains with a conic point. Sibirsk. Mat. Zh., 29-2, 1988.

[26] V. A. Kozlov, V. G. Maz'ya, J. Rossmann. Spectral properties of operator pencils generated by elliptic boundary value problems for the Lamé system. Rostock. Math. Kolloq., $\mathbf{5 1}, 1997,5-24$.

[27] V. A. Kozlov, V. G. Maz’ya, J. Rossmann. Conic singularities of solutions problems in hydrodynamics of a viscous fluid with a free surface. Matematica Scandinavia. To appear, 1998 .

[28] V. A. Kozlov, V. G. Maz'Ya, C. Schwab. On singularities of solutions of the displacement problem of linear elasticity near the vertex of a cone. Arch. Rational Mech. Anal., 119, 1992, $197-227$.

[29] V. A. Kozlov, V. G. Maz'ya, C. Schwab. On singularities of solutions of the Dirichlet problem of hydrodynamics near the vertex of a cone. J. reine angew. Math., 456, 1994, 65-97. 
[30] G. Lebeau. Propagation des ondes dans les variétés à coins. Ann. Sci. École Norm. Sup., 30-4, 1997, 429-497.

[31] D. Legulllon. Computation of 3D-singularities in elasticity. In M. Costabel, M. Dauge, S. NiCAISE, editors, Boundary value problems and integral equations in nonsmooth domains (Luminy, 1993), Lecture Notes in Pure and Appl. Math., 167, Marcel Dekker, Inc., New York, 1995, 161-170.

[32] R. Lozi. Résultats numériques de régularité du problème de Stokes et du laplacien itéré dans un polygone. RAIRO Analyse Numérique, 12-3, 1978, 267-282.

[33] V. G. Maz'ya, S. A. Nazarov, B. A. Plamenevskit. Asymptotische Theorie elliptischer Randwertaufgaben in singulär gestörten Gebieten. Mathematische Monographien, Band 82 \& 83, Akademie Verlag, Berlin, 1991.

[34] V. G. Maz'ya, B. A. Plamenevskit. $L^{p}$ estimates of solutions of elliptic boundary value problems in a domain with edges. Trans. Moscow Math. Soc., 1, 1980, 49-97.

[35] V. G. Maz’ya, B. A. Plamenevskit. Estimates in $L^{p}$ and in Hölder classes and the MirandaAgmon maximum principle for solutions of elliptic boundary value problems in domains with singular points on the boundary. Amer. Math. Soc. Transl, 123-2, 1984, 1-56.

[36] V. G. Maz’ya, J. Rossmann. Über die Asymptotik der Lösungen elliptischer Randwertaufgaben in der Umgebung von Kanten. Math. Nachr., 138, 1988, 27-53.

[37] S. A. Nazarov. Asymptotic behavior of the solution of the Neumann problem at a point of tangency of smooth components of the domain boundary. Izv. Ross. Akad. Nauk Ser. Mat., 58-1, 1994, 92-120.

[38] S. A. Nazarov. On the water flow under a lying stone. Mat. Sb., 186-11, 1995, 75-110.

[39] S. A. Nazarov, B. A. Plamenevskit. Elliptic Problems in Domains with Piecewise Smooth Boundaries. Expositions in Mathematics,13, Walter de Gruyter, Berlin, 1994.

[40] S. A. Nazarov, O. R. Polyakova. Fracture criteria asymptotic conditions at crack tips, and selfadjoint extensions of the Lamé operator. Tr. Mosk. Mat. Obs., 57, 1996, 16-74.

[41] S. NiCaise. Polygonal interface problems. Methoden und Verfahren der Mathematischen Physik, 39, Verlag Peter D. Lang, Frankfurt-am-Main, 1993.

[42] S. Nicaise, A. M. SÄndig. General interface problems I/II. Math. Meth. in the Appl.Sci, 17, 1994, 395-450.

[43] M. Orlt, A. M. SÄndig. Regularity of Navier-Stokes flows in nonsmooth domains. In M. Costabel, M. Dauge, S. Nicaise, editors, Boundary Value Problems and Integral Equations in Nonsmooth Domains, Lecture Notes in Pure and Applied Mathematics, 167, Marcel Dekker, Inc., New York, 1994, 185-201.

[44] G. Rauget. Résolution numérique par une méthode d'éléments finis du problème de Dirichlet pour le laplacien dans un polygone. C. R. Acad. Sci. Paris, Sér. I, 286-18, 1978, 791-794.

[45] H. Schmitz, K. Volk, W. Wendland. Three-dimensional singularities of elastic fields near vertices. Numer. Methods Partial Differential Equations, 9-3, 1993, 323-337.

[46] E. Stephan, J. R. Whiteman. Singularities of the Laplacian at corners and edges of threedimensional domains and their treatment with finite element methods. Math. Methods Appl. Sci., 10-3, 1988, 339-350. 
[47] B. A. Szabó, Z. Yosibash. Numerical analysis of singularities in two dimensions. I. Computation of eigenpairs. Internat. J. Numer. Methods Engrg., 38-12, 1995, 2055-2082.

[48] B. A. Szabó, Z. Yosibash. Numerical analysis of singularities in two dimensions. II. Computation of generalized flux/stress intensity factors. Internat. J. Numer. Methods Engrg., 39-3, 1996, 409-434.

Slides and the list of references "Corner and Edge Singularities: A Closer Look at Parameter Dependence and Variable Coefficients", (1997), by M. Costabel can be retrieved from the web http://www.maths.univ-rennes1.fr/ " costabel/ .

Institut Mathématique de Rennes, UMR-CNRS 6625,

Université de Rennes 1, Campus de Beaulieu, 35042 Rennes Cedex

http://www.maths.univ-rennes1.fr/ dauge 